\title{
On the dust content of galaxy clusters
}

\author{
C. M. Gutiérrez ${ }^{1,2}$ and M. López-Corredoira ${ }^{1,2}$ \\ 1 Instituto de Astrofísica de Canarias, 38205 La Laguna, Tenerife, Spain \\ e-mail: cgc@ll.iac.es \\ 2 Departamento de Astrofísica, Universidad de La Laguna, 38206 La Laguna, Tenerife, Spain
}

Received 14 July 2014 / Accepted 5 August 2014

\begin{abstract}
Context. Most of the contribution to dust emission in clusters of galaxies comes from late-type galaxies. However, several ejection processes of material from these galaxies could introduce dust in the intracluster media. Even a relatively low abundance of this dust could act as an efficient cooling agent and have a relevant role in the evolution of clusters.

Aims. We present a study to estimate the dust content in galaxy clusters.

Methods. This was done by using one the most complete existing catalogues of galaxy clusters based on Sloan Digital Sky Survey (SDSS) data and following two methods: the first one compares the colours of samples of galaxies in the background of clusters with those of galaxies in the field. Using this method, we have explored clustercentric distances up to $6 \mathrm{Mpc}$; this covers at least $2 \times R_{200}$ for all the clusters in the sample. The galaxies used in this first method were selected from the SDSS-DR9, among those having reliable photometry and accurate estimation of photometric redshifts. Using the colours of background galaxies, we analyzed several regions at galactic latitudes $|b|>20^{\circ}$ and $>50^{\circ}$. The results are largely independent of the galactic cut applied. At $|b|>20^{\circ}$, the sample contains 56985 clusters in the redshift range $0.05<z<0.68$ (the mean redshift is 0.30 ) and $\sim 5.3 \times 10^{6}$ galaxies. The second method computes the contribution of dust in clusters of galaxies to the far infrared sky. That is estimated indirectly by measuring the effect of clusters in the $E(B-V)$ extinction map.

Results. Using the first method, we did not find any dependence with clustercentric distance in the colours of background galaxies. As representative of the whole results, the surface integral of the excess of colour $g-i$ in three rings centred in the clusters and with radius $0-1,0-2$, and $0-3 \mathrm{Mpc}$ is $-3.7 \pm 3.5,+3.2 \pm 6.8$, and $-4.5 \pm 10.1$ milimag $\mathrm{Mpc}^{2}$, respectively. This allows us to constrain the mass of dust in the intracluster media, $M_{\text {dust }}<8.4 \times 10^{9} M_{\odot}(95 \%$ C.L.) within a cluster radius of $3 \mathrm{Mpc}$. With the second method, which averages the extinction of all clusters, we find a surface integral of the excess of colour $g-i$ of $3.4 \pm 0.1 \mathrm{millimag} \mathrm{Mpc}^{2}$. From the extinction and redshift of each cluster, we obtain $0.13 \mathrm{Jy}$ and $(1.46 \pm 0.03) \times 10^{45} \mathrm{erg} \mathrm{s}^{-1}$ for the mean flux and luminosity at $100 \mu \mathrm{m}$. This is $\sim 60$ times the far infrared luminosity of a Milky Way-like galaxy. By assumming similar properties for the dust, we can estimate a total dust mass per cluster of $\sim 2 \times 10^{9} M_{\odot}$, which is compatible with the hypothesis that the dust is within the spiral galaxies of a cluster. Separating the clusters in $5 \times 5$ bins in redshift and richness, we confirm previous findings of a clear increase in luminosity with a redshift that agrees with the trend expected from current models.

Conclusions. The results are compatible with expectations accounting for the contribution of dust in cluster member galaxies and, in particular, a strong increase in luminosity with redshift is demonstrated. The dust in the intracluster media is not detected, but the analyses as a whole show that the amount of this dust is small.
\end{abstract}

Key words. galaxies: clusters: general - galaxies: clusters: intracluster medium

\section{Introduction}

Intergalactic media in galaxy clusters does not offer a comfortable ambient for dust grains to survive. Dust grains are progressively destroyed by the collision of particles (Draine \& Salpeter 1979). Although this process is relatively fast, the exact sputtering time depends on the density and temperature of the media, as well as on the chemical composition and size of the grains. For a wide range in temperature, the growth of dust by accretion is not enough to compensate the sputtering, although several mechanisms like mergers, supernova winds, or ram-pressure stripping can inject dust continuously into intergalactic regions of clusters. Little is known from an observational point of view; several pioneer studies (Zwicky 1962; Karanchetsev \& Lipovetskii 1969; Bogart \& Wagoner 1973; Boyle et al. 1988; Romani \& Maoz 1992) compared the attenuation of the light as a function of wavelength in objects situated in the background of clusters with those of objects in the field. Using single cases or small samples of clusters, extinctions within the range $\sim 0.1-0.4$ mag were estimated. A huge advance was made by Maoz (1995), who recognized the existence of selection effects in some of these previous studies and put a constraint $<0.05 \mathrm{mag}$ on the excess of colour of the light of background galaxies crossing foreground Abell clusters. Xilouris et al. (2006) discover a systematic shift in the colour of background galaxies viewed through the intergalactic medium of the nearby M81 group. This reddening coincides with atomic, neutral gas that is previously detected between the group members. Myers et al. (2003) find anticorrelation between quasi stellar objects (QSOs) and clusters but little reddening. They suggest gravitational lensing as a possible explanation of the anticorrelation. In the last decade, several groups have used large compilations of data that are mostly Sloan Digital Sky Survey (SDSS) based to put much tighter constraints. Essentially, all of them used a common technique based on the attenuation and reddening of the light of background objects (galaxies and/or QSOs) when passing through a cluster. This is quantified by 
comparing the same properties of objects in the field. Nollenberg et al. (2003) used nearby, medium, and poor APM ${ }^{1}$ galaxy clusters and derived a $99 \%$ C.L. upper limit in the reddening due to dust in crossing clusters $A_{R}=0.025 \mathrm{mag}$ on $1.3 \mathrm{Mpc}$ scales. Chelouche et al. (2007) compared the photometric and spectroscopic properties of quasars behind clusters with those in the field. By using the SDSS-DR5 spectroscopic quasar sample (Schneider et al. 2007) and the catalogue of SDSS clusters obtained by Koester et al. (2007), they detected an excess of colour $E(B-V) \sim 10^{-3} \mathrm{mag}$ on Mpc scales. Bovy et al. (2008) used a sample of SDSS luminous, early-type galaxies and the SDSS cluster catalogue obtained by Berlind et al. (2006) and obtained restrictions $E(B-V)<3 \times 10^{-3}$ and $8 \times 10^{-3}$ mag on scales of $1-2$ and $<1 \mathrm{Mpc}$, respectively. Muller et al. (2008), using a catalogue of 485 clusters and galaxies extracted from the Red-sequence Cluster Survey (Gladders \& Yee 2005) and a catalogue of 90000 galaxies with photometric redshifts in the range $0.5-0.8$, did not find evidence of colour differences with clustercentric distance and put a severe restriction on the average visual extinction of $\left\langle A_{v}\right\rangle=0.004 \pm 0.010 \mathrm{mag}$ within a clustercentric distance $R_{200}$. McGee \& Balogh (2010) explored the presence of dust on large scales by using 70000 low redshift SDSS galaxy groups and clusters. They claim the detection of dust out to a cluster-centric distance of $30 \mathrm{Mpc} h^{-1}$. Muller et al. (2009) summarized the results of those studies (see their Table 1).

Other authors had followed a more direct approach searching for the contribution of intracluster dust emission to the far infrared sky maps. Of course, the total IR emission of the cluster contains the contribution from dust within galaxy members and within the intracluster media. First claims of direct extended spatial detections (Wise et al. 1993) in the infrared using IRAS were controversial with several authors (e.g. Stickel et al. 2002) pointing out that part of these detections could be due to dust within galaxy members of the clusters, or to Galactic cirrus. The most complete study following this method was conducted by Montier \& Giard (2005) and Giard et al. (2008) combining IRAS data of more than 10000 clusters. Those authors obtained a clear statistical detection of emission in the bands at 12, 25, 60, and $100 \mu \mathrm{m}$. According to the estimation of IR emission due to the different galaxy populations in cluster member galaxies, Roncarelli et al. (2010) concluded that most (if not all) of the signal detected comes from the emission of dust in cluster members. The MIPS (Multiband Imaging Photometer for Spitzer) offered a significant quantitative improvement that has allowed studies of single clusters (Bai et al. 2007 on Abell 2029; and Kitayama et al. 2009 on Coma cluster). These last authors put the following constraints on the emission of dust within the central $100 \mathrm{kpc}: \sim 5 \times 10^{-3}$, $6 \times 10^{-2}$, and $7 \times 10^{-2} \mathrm{MJy} \mathrm{s}^{-1}$ at 24, 70 and $160 \mu \mathrm{m}$ respectively. Following Kitayama et al., this translates to an expected visual extinction $A_{v}<0.02 \mathrm{mag}$ and a surface mass density of dust of $\Sigma_{\mathrm{d}}<1.4 \times 10^{3} M_{\odot} \mathrm{kpc}^{-2}$.

The study presented here combines and extends the two approaches outlined above. In both cases, we follow a statistical approach averaging the contribution of a large sample of clusters and background galaxies selected from the SDSS survey. The paper is structured as follows: After this introduction, Sect. 2 describes the properties of the samples used, the restrictions applied to get the final subsamples, and the methology; Sect. 3 analyzes the main results, estimates the amount of dust per cluster, and compares it with the results presented by other authors and with theoretical expectations; conclusions are presented in Sect. 4

\footnotetext{
1 APM: Automated Plate Measuring machine.
}

\section{Samples and methodology}

As outlined in the previous section, we follow two methods: in the first, we study the additional reddening of galaxies in the background of clusters as compared with similar galaxies in the field; in the second, we estimate the contribution of clusters of galaxies to the optical extinction maps. The basic ingredients of our study are a map of extinction and catalogues of clusters and galaxies, respectively. The sample of galaxies was obtained from the SDSS DR9 photometric catalogue (Ahn et al. 2012). Several potential cluster catalogues were considered. On the basis to optimize completitude and sky and redshift coverage, the sample of clusters obtained by Wen et al. (2012) was chosen using the SDSS-III survey. The spatial coverage of that survey is $\sim 14000$ square degrees and contains 132684 clusters in the redshift range $0.05 \leq z \leq 0.8$. According to those authors, the catalogue is more than $95 \%$ complete for clusters with a mass of $M_{200}>10^{14} M_{\odot}$ in the range $0.05 \leq z \leq 0.42$ and contains a false detection rate less than $6 \%$. The catalogue presents photometric redshifts for all the clusters, whilst only $\sim 30 \%$ have determination of redshift based on the spectroscopy of their brighter cluster galaxy (an updated version of the catalogue with 52683 spectroscopic redshifts was presented by Wen $\&$ Han 2013). From these cases, it was estimated for the photometric estimation of redshifts, a systematic offset $<0.004$ and a standard deviation $<0.018$. To complete the studies to lower redshifts, we also use the sample of 1059 Abell clusters with spectroscopic redshifts ${ }^{2}$.

The map for extinction used was the well known and widely used map by Schlegel et al. (1998). This map covers the full sky and presents an estimation of the $E(B-V)$ extinction obtained from the analysis of COBE DIRBE and IRAS data. The pixel size is 2.37 arcmin with a $F W H M=6.1$ arcmin. Although the inmense majority of the signal in that map is due to the Galactic extinction, any contribution from the dust of extragalactic objects obviously leaves its own imprint ${ }^{3}$. According to such authors, the maximum level of such possible extragalactic contamination is below $0.01 \mathrm{mag}$ and should be nearly uniformly distributed.

\subsection{Method 1}

For each of the galaxies from SDSS-DR9, we downloaded the relevant information for this study: equatorial coordinates, magnitudes in the $g, r$, and $i$ filters, photometric redshifts, and the corresponding errors as estimated by SDSS pipelines ${ }^{4}$. The number of objects catalogued as galaxies in that survey and having clean photometry ${ }^{5}$ is $\sim 1.34 \times 10^{8}$. We selected those galaxies with photometric errors $<0.1 \mathrm{mag}$ and consider a cut of $22 \mathrm{mag}$ in the three filters, $g, r$, and $i$, considered. This dramatically reduces the sample to $31,182,824$ objects. We also removed a few outliers $(\sim 0.07 \%$ of the sample) with extreme $g-r(<-0.5$ or $>2.6 \mathrm{mag})$ or $g-i(<0.0$ or $>3.9 \mathrm{mag})$ colours.

\footnotetext{
2 See http://heasarc.gsfc.nasa.gov/W3Browse/ galaxy-catalog/abellzcat.html

3 Only the contribution from extragalactic objects with flux $>1.2 \mathrm{Jy}$ had been removed by Schlegel et al.

4 From the various estimations of magnitudes computed by SDSS pipeline, we follow the prescription in SDSS web pages and used the magnitudes called modelmag. SDSS computes photometric redshifts according to several algorithms; we used the one recorded in the table Photoz (for details see SDSS web pages).

5 See SDSS web pages for an explanation of this concept.
} 
As it is usual in extragalactic studies, we ignore the region close to the Milky Way plane $(|b|<20$ degrees) where any possible effect of reddening by clusters would be largely masked by the heavy Galactic extinction at low latitudes. The exact cut in latitude is a compromise to select those regions with low Galactic extinction, whilst at the same time maintaining a sample of clusters large enough. We ran numerous tests changing the Galactic latitude cut within the range 20-50 degrees, and in addition, we removed those clusters from the analysis situated in regions with mean extinctions $>0.05$ mag as measured in circle with a radius of $6 \mathrm{Mpc}$ projected at the cluster distance. Nevertheless, none of the results presented here is critically dependent from those restrictions.

The following step was to determine the relative position of each galaxy with respect to the sample of clusters. For clusters, we use a conservative error in the photometric redshift of 0.02 (see Wen et al. 2012), whilst we use the corresponding errors listed in the SDSS catalogue for galaxies. For each cluster, we selected all galaxies that are projected at clustercentric distances up to $6 \mathrm{Mpc}$. This distance is $>2 \times R_{200}$ for all the clusters in our sample. The uncertainty in the estimation of such distance due to the uncertainty in the photometric redshift of the clusters are $\sim 30,20$, and $8 \%$ at redshifts $0.05,0.1$ and 0.2 , respectively, so this could introduce some filtering in the case of a radial dependence of colours with clustercentric distance.

A galaxy was considered to be in the background of a cluster when $\Delta z \equiv z_{\text {gal }}-z_{\text {cluster }}>3 \sqrt{\sigma_{z_{\text {gal }}}^{2}-\sigma_{z_{\text {cluster }}}^{2}}$. The relatively large error in redshift, due to the photometric technique used for the determination of the redshifts in the galaxies and clusters, makes it impossible to know their relative position in the space of redshift for most $(\sim 2 / 3)$ of the galaxies projected along the line of sight of a given cluster.

Using a cut in galactic latitude of 20 degrees, the number of clusters and background galaxies selected by this method are 56985 and 5287825 (the corresponding numbers at $|b|>50$ degrees are 36473 and 3520658 , respectively). Most of these galaxies behind a given cluster are projected in the background of more than one cluster; they cross 2.1 clusters at projected clustercentric distances $<6 \mathrm{Mpc}$ on average. Figure 1 presents the redshift distribution of clusters and the mean number of background galaxies as a function of the redshift of the cluster. The cluster distribution extends from redshift 0.05 to 0.6 , having the maximum at $\sim 0.25$. This does not exactly reproduce the distribution of redshifts in the Wen et al. (2012) catalogue, as clusters at very high redshift have not been considered because they do not have background objects in the galaxy sample. As expected, the number of galaxies projected behind a given cluster depends strongly on the redshift of the cluster; this is basically a consequence of the cut in magnitude adopted for the galaxy sample.

Maps of $(g-r),(g-i)$ and $(r-i)$ colours and the corresponding radial profiles are shown in Figs. 2 and 3, respectively. These maps represent the mean colours of galaxies in the background of clusters as a function of the projected clustercentric distance. The radius of the maps and the binning are $6 \mathrm{Mpc}$ and $0.3 \mathrm{Mpc} \times 0.3 \mathrm{Mpc}$, respectively. The mean values of the colours are $1.348 \pm 0.004,1.905 \pm 0.006$, and $0.557 \pm 0.003$ for $g-r$, $g-i$, and $r-i$, respectively. If we select only those objects at $|b|>50$ degrees, the results are similar with a slightly larger dispersion in each colour. None of the maps show any evidence of gradient or structure. The limits on the excess of colours as a function of clustercentric distance were estimated by taking a ring centred in each cluster as reference and having inner and outer radii of 4 and $5.5 \mathrm{Mpc}$, respectively. The error bars were

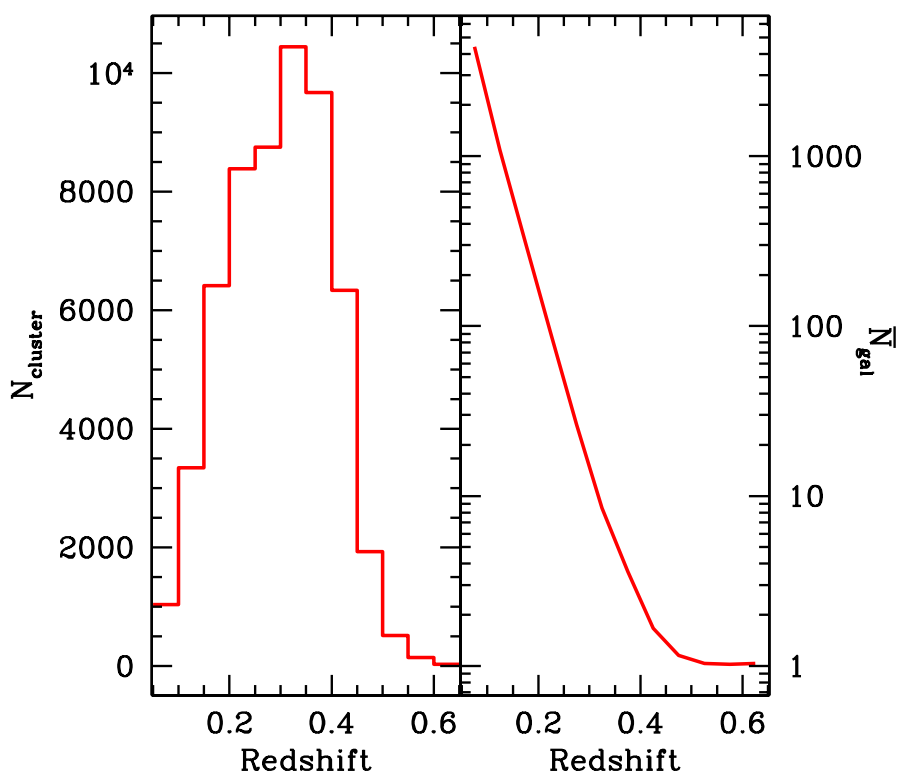

Fig. 1. Left: histogram of number of clusters as a function of redshift. Right: mean number of galaxies in the background of clusters.

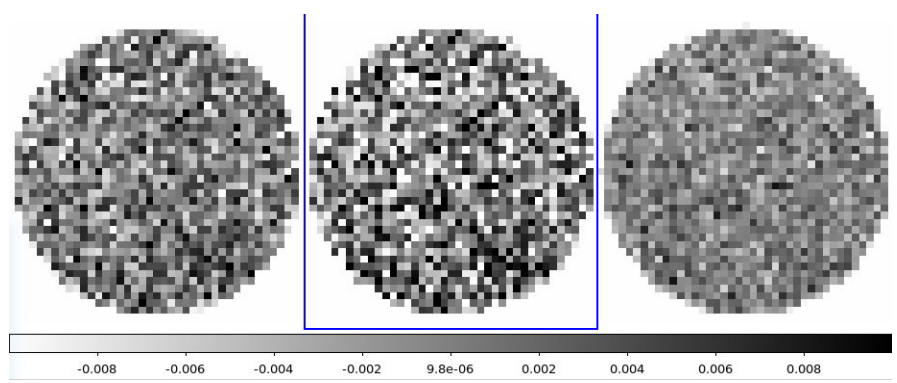

Fig. 2. Map of colours $g-r, g-i$, and $r-i$ (from left to right respectively) of mean colours of galaxies as a function of the projected clustercentric distance. The maps have a radius of $6 \mathrm{Mpc}$ and a binning of $0.3 \times 0.3 \mathrm{Mpc}^{2}$. The mean colour of each map has been subtracted. The gray scale is linear and has an amplitude of $0.02 \mathrm{mag}$.

Table 1. Average excess of colours (in milimags $\mathrm{Mpc}^{2}$ ) within different clustercentric radius.

\begin{tabular}{cccc}
\hline \hline $\operatorname{Rad}(\mathrm{Mpc})$ & $\Delta(g-r)$ & $\Delta(g-i)$ & $\Delta(r-i)$ \\
\hline $0-1$ & $-2.23 \pm 2.46$ & $-3.67 \pm 3.52$ & $-1.43 \pm 1.53$ \\
$0-2$ & $+1.54 \pm 4.73$ & $+3.20 \pm 6.77$ & $+1.66 \pm 2.94$ \\
$0-3$ & $-4.73 \pm 7.06$ & $-4.52 \pm 10.10$ & $+0.21 \pm 4.39$ \\
\hline
\end{tabular}

computed from the dispersion of colours in a given bin and contain the uncertainty in the estimation of the mean galactic extinction in addition to the uncertainty in the estimation of the mean colour. The results within three regions centred in the clusters are shown in Table 1. We do not detect excess of colours within any of the three radii considered. These results allow us to put 95\% C.L. upper limits for the colour excess within a circle with radius $1 \mathrm{Mpc}$ centred in the clusters of 2.69, 3.37, and 1.63 milimags $\mathrm{Mpc}^{2}$ in $(g-r),(g-i)$ and $(r-i)$, respectively.

The above procedure introduces some degree of filtering in the reddening by each cluster due to the fact that a given galaxy could be in the background of more than one cluster, and then its light crosses each foreground cluster at different clustercentric distances. The amount of filtering would depend on the unknown spatial distribution of intracluster dust. To avoid this uncertainty, 


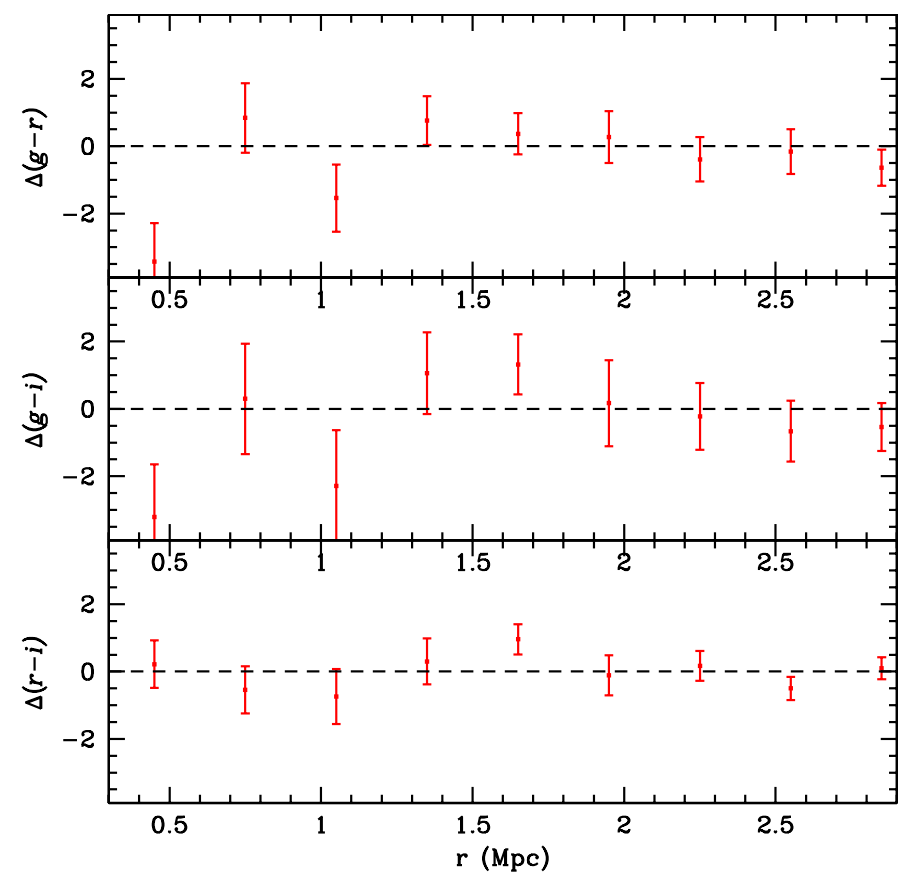

Fig. 3. Excess of colours as a function of projected clustercentric distance. The units of the vertical axis are milimags.

several tests were conducted by modelling the dust spatial distribution. Here, we only give the results of a very conservative approach that avoids any a priori dust modelling by considering only those galaxies lying in the background of just one cluster. In doing that, the size of the sample drastically reduces to $\sim 7000$ clusters and $1.8 \times 10^{5}$ galaxies. We also do not detect any excess of signal within any clustercentric distance. The corresponding 95\% C.L. limits are 120, 39, and 40 milimags $\mathrm{Mpc}^{2}$ for the excess of colours $g-r, g-i$ and $r-i$ in a projected clustercentric radius of $1 \mathrm{Mpc}$, respectively.

\subsection{Method 2}

We follow the method first proposed by Kelly \& Rieke (1990) and developed by Montier \& Giard (2005). As discussed by these authors (an references therein), the detectability of individual clusters in IRAS infrared maps is compromised by the low sensitivity of the maps, as compared to the signal expected from clusters and to the noise confusion level of $\sim 1 \mathrm{MJy} / \mathrm{sr}$ due to extragalactic sources in those maps. Instead of using the infrared maps as Montier \& Giard did, we used the extinction map $E(B-V)$ by Schlegel et al. (1998). Both approachs are similar as the extinction $E(B-V)$ map is basically the point-source subtracted IRAS $100 \mu \mathrm{m}$ map, corrected to a reference temperature of $18.2 \mathrm{~K}$ using the DIRBE temperature map, and multiplied by a constant $p=0.0184 \pm 0.0014 \mathrm{mag} /\left(\mathrm{MJy} \mathrm{sr}^{-1}\right)$. The estimations obtained using this method contain the contribution of dust in the ICM and within galaxy members.

We built a $2 \times 2 \mathrm{deg}$ (this corresponds to a radius $>3 \mathrm{Mpc}$ for all the clusters in the Wen et al. sample) $E(B-V)$ map in galactic coordinates centred on each cluster, each rotated by a random angle multiple of $\pi / 2$, and averaged all the maps. Given the large spatial density of clusters, a given pixel in the extinction maps might contribute to several cluster maps. This introduces some degree of correlation between pixels. After many tests, we decided to limit the analysis to those clusters at a galactic latitude $|b|>50 \mathrm{deg}$. Although the mean galactic extinction

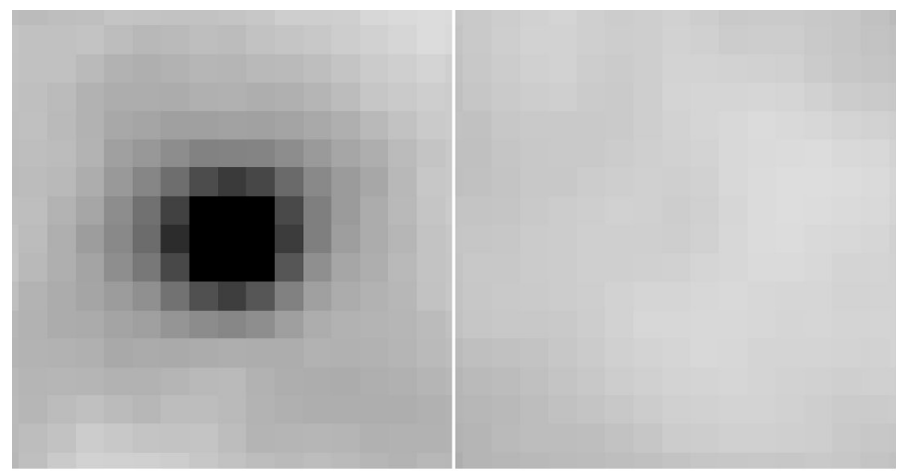

Fig. 4. Left: map of extinction $(48 \times 48$ arcmin) obtained by averaging the maps centred on each cluster. Right: a simulated map obtained shifting the position of each cluster an angle randomly distributed in the range 1-2 degrees.

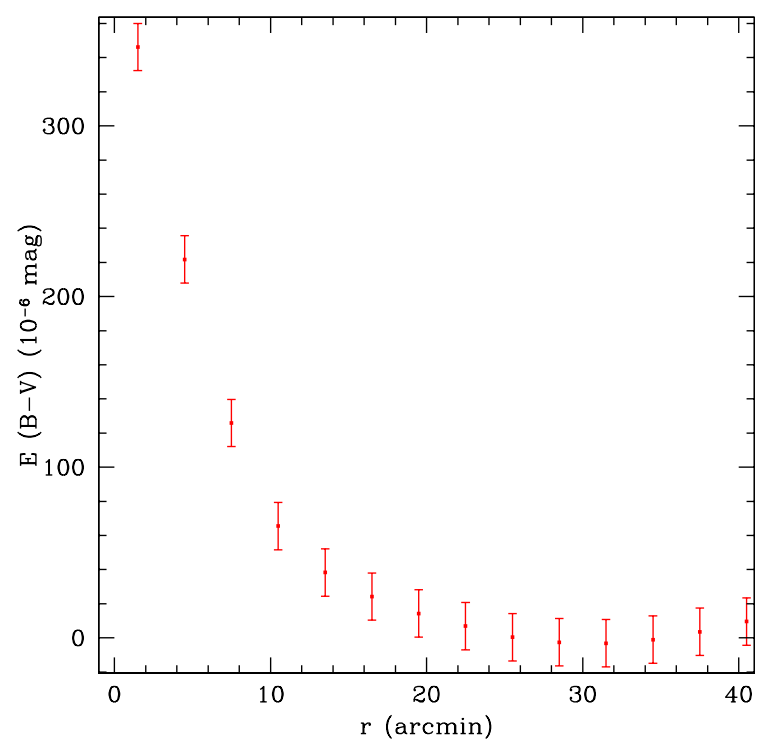

Fig. 5. Radial profile of the mean extinction in the stacked maps shown in previous figure. The contribution of the Galactic extinction has been removed by averaging a ring of inner and outer radius of 24 and 40 arcmin respectively.

is 0.024 mag at $|b|>50 \mathrm{deg}$, we still flagged out all clusters situated in regions of particularly heavy Galactic extinction, or those immersed in large gradients, i.e. those with a mean level of extinction $>0.05 \mathrm{mag}$ or a $\mathrm{rms}>0.01 \mathrm{mag}$ within a square of $2 \times 2$ degrees centred in the cluster. None of the results presented in the paper depends on the specific values of such constraints. The final sample contains 52667 clusters.

Figure 4 presents the average extinction map; this shows a clear signal concentric with the position of the clusters. The radial profile of the extinction map is shown in Fig. 5; this indicates an extinction at the centre of the clusters $E(B-V)=$ $346 \times 10^{-6} \mathrm{mag}$. To assess the reliability of the signal and the absence of significant systematics, we have created a new stacked map by randomly and independently shifting the position of each cluster by an angle uniformly distributed in the range 12 degrees in galactic longitude. The resulting map is shown in Fig. 4 and is compatible with noise random fluctuations. From the uncertainties in the estimation of the extinction from our own Galaxy and from the simulated results, we estimate a conservative uncertainty in the central value of $\sim 50 \times 10^{-3}$ mag; the main factor contributing to that is the absolute level of the residual 
extinction from the Milky Way. The extinction has a Gaussian profile width of $F W H M=12$ arcmin. Applying the conversion factor between $E(B-V)$ extinction and emission at $100 \mu \mathrm{m}$ quoted in Schlegel et al., the central emission translates into a mean emission at $100 \mu \mathrm{m}$ of $(1.88 \pm 0.08) \times 10^{4} \mathrm{Jy} \mathrm{sr}^{-1}$. As it is demonstrated by separating the clusters in bins of different richnesss and redshifts, this profile does not reflect the spatial distribution of the cluster, but it is instead dominated by the instrumental resolution of the extinction map and possibly additional filtering due to the method.

\section{Analysis}

\subsection{Method 1: Constraints on intracluster dust content}

For the case of a Milky Way-like dust, in which $A_{V}=1.73 \Delta(\mathrm{g}-$

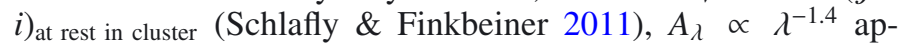
proximately in the interval from $g$ to $i$ filters. Hence, $\Delta(g-$ $i)_{\text {at rest in cluster }} \approx \Delta(g-i)_{\text {obs }}\left(1+z_{\text {cluster }}\right)^{-1.4}$. Since

$\int A_{V} \mathrm{~d} S=\int \mathrm{d} V a_{V}$

$a_{V}(r)=1.086 \kappa_{V} \rho_{\text {dust }}(r)$,

$M_{\text {dust }}=\int \mathrm{d} V \rho_{\text {dust }}(r)$,

the amount of dust within a given radius can be constrained using

$\frac{M_{\text {dust }}(r<R)}{M_{\odot}}=\frac{4.4 \times 10^{15}}{\kappa_{v}} \int_{0}^{R} A_{v} \mathrm{~d} S$,

where $\kappa_{v}=1.33 \times 10^{4} \mathrm{~g} \mathrm{~cm}^{-2}$ (Mathis 1990; Loeb \& Haiman $1997)$ and $S$ is the projected area in $\mathrm{Mpc}^{2}$. From the values quoted in Table 1 for the excess $\Delta(g-i)$ within clustercentric distances of 1,2 and $3 \mathrm{Mpc}$, we obtained the corresponding $\int_{0}^{R} A_{v} \mathrm{~d} S$ values (see above) and estimated $95 \%$ C.L. limits on the mass of intracluster dust of $1.8 \times 10^{9}, 9.0 \times 10^{9}$, and $8.4 \times 10^{9} M_{\odot}$ respectively. This corresponds to a limit on the projected density of dust of $\Sigma<573,717$ and $297 M_{\odot} \mathrm{kpc}^{-2}$ within the clustercentric radius of $1,2,3 \mathrm{Mpc}$, respectively.

Assuming that our Galaxy has a dust exponential disc with scalelength $2.2 \mathrm{kpc}$ (Drimmel \& Spergel 2001), except in the inner $4 \mathrm{kpc}$ to take the inner hole into account (López-Corredoira et al. 2004), and that the extinction observed in the Galactic poles is $A_{V}=0.05 \mathrm{mag}$ (Schlegel et al. 1998), the Milky Way would produce $8.6 \times 10^{-5} \mathrm{mag} \mathrm{Mpc}^{2}$ observed from outside, which is around a dust mass of $2.8 \times 10^{7} M_{\odot}$ (from Eq. (1)). Davies et al. (1997), who used far-infrared emission, gets a similar number: $M_{\text {dust }}=3 \times 10^{7} M_{\odot}$. This means that we are constrained to have less than 300 times (95\% C.L.) the dust mass of the Milky Way in the intracluster medium.

\subsection{Method 2: Infrared luminosities and total dust content}

The flux at rest is calculated as $F_{v, \text { rest }}=(1+z)^{-1-\alpha} F_{v, \text { observed }}$, given that the emission $I_{v} \propto v^{\alpha}$; we take $\alpha=2$ (Schlegel et al. 1998). The extra factor $(1+z)^{-1}$ stems from the increase of the frequency range, which gives an observed flux larger by a factor $(1+z)$. The mean luminosity at $100 \mu \mathrm{m}$ is $(1.46 \pm 0.03) \times 10^{45} \mathrm{erg} \mathrm{s}^{-1}$ for the whole sample. The luminosity produced by the cold dust component of the Milky Way is
$2.1 \times 10^{43} \mathrm{erg} \mathrm{s}^{-1}$ (Cox et al. 1986) or $2.6 \times 10^{43} \mathrm{erg} \mathrm{s}^{-1}$, according to Davies et al. (1997). Assuming that the emission at $100 \mu \mathrm{m}$ includes most of the dust emission in clusters, the value quoted above would correspond to the emission of $\sim 60$ Milky Way galaxies which is of the order of the number of spiral galaxies found in clusters, which is $M_{\text {dust }} \sim 2 \times 10^{9} M_{\odot}$ following the numbers of the previous subsection.

To compare the results of method 1 given in Table 1, we can convert the luminosity into the equivalent surface integral of the associated absorption by dust:

$$
\int \mathrm{d} S A_{V}=3.1 \pi R^{2} \overline{E(B-V)}
$$

$\overline{E(B-V)}=0.0184 \mathrm{mag} /(\mathrm{MJy} / \mathrm{sr})=\frac{F_{100, \text { rest }}}{\pi \alpha^{2}}, \alpha=\frac{R}{d_{\mathrm{A}}}$,

$v F_{100, \text { rest }}=\frac{L_{100, \text { rest }}}{4 \pi d_{\mathrm{L}}^{2}}$,

where $d_{\mathrm{A}}$ is the angular distance, $d_{\mathrm{L}}$ is the luminosity distance, which are related by $d_{\mathrm{A}}=\frac{d_{\mathrm{L}}}{(1+z)^{2}}$. Hence,

$\int \mathrm{d} S A_{V}=1.58 \times 10^{-47} \frac{L_{100, \text { rest }}(\mathrm{erg} / \mathrm{s})}{\left\langle(1+z)^{4}\right\rangle}$,

which gives us $\int \mathrm{d} S A_{V}=(5.9 \pm 0.1)$ millimag $\mathrm{Mpc}^{2}$, or $\int \mathrm{d} S \Delta(g-i)=(3.4 \pm 0.1)$ millimag $\mathrm{Mpc}^{2}$ with the above number of $L_{100 \text {,rest }}(\mathrm{erg} / \mathrm{s})=(1.46 \pm 0.03) \times 10^{45}$ and a value for our sample of clusters of $\left\langle(1+z)^{4}\right\rangle=3.87$. Using Eq. (2), we get again a dust mass of $2 \times 10^{9} M_{\odot}$.

According to the discussion by Roncarelli et al. (2010), the infrared emission at $100 \mu \mathrm{m}$ from galaxies in clusters is dominated by the emission of late-type spiral galaxies. Those authors estimated a flux of $1904.8_{-429.1}^{+617.1} \mathrm{Jy}$ in a sample of 7476 MaxcBCG clusters (Koester et al. 2007), or the value of $0.255_{-0.057}^{+0.083} \mathrm{Jy}$ per cluster (we estimate a mean flux per cluster of $0.130 \pm 0.005 \mathrm{Jy}$ ), and concluded that the values detected by Giard et al. (2008) are roughly compatible with their estimations and leave little chance for any component associated to intracluster dust. Our estimations agree with those by Giard et al. (see Sect. 3.4) and then reinforces that scenario. The limit found by estimating the possible reddening galaxies behind clusters (Method 1), although not very tight, are compatible with this. The detection of intracluster dust using this method would require a larger sample of background objects.

\subsection{Abell clusters}

As a test of the consistency of our results and to study the range in redshift $z<0.05$ that is uncovered by the Wen et al. (2012) sample, we do a similar analysis for the sample of 1059 Abell clusters with measured redshifts ${ }^{6}$. Considering the whole sample and applying similar restrictions in galactic latitude and extinctions as to the Wen et al. sample, we selected 485 clusters. The average extinction map (Fig. 6) centred on each cluster shows a clear excess of signal with a central peak amplitude of $E(B-V)=(1.16 \pm 0.14) \times 10^{-3} \mathrm{mag}$ that corresponds to a density of flux $\sim(6.3 \pm 0.8) \times 10^{4} \mathrm{Jy} \mathrm{sr}^{-1}$. The radial profile has a $F W H M \sim 14$ arcmin, which integrates up to a radius of 21 arcmin in which we obtain a $100 \mu \mathrm{m}$ luminosity of $(0.79 \pm 0.10) \times 10^{45} \mathrm{erg} \mathrm{s}^{-1}$. If we restrict the analysis to those

\footnotetext{
6 See http://heasarc.gsfc.nasa.gov/W3Browse/ galaxy-catalog/abellzcat.html
} 

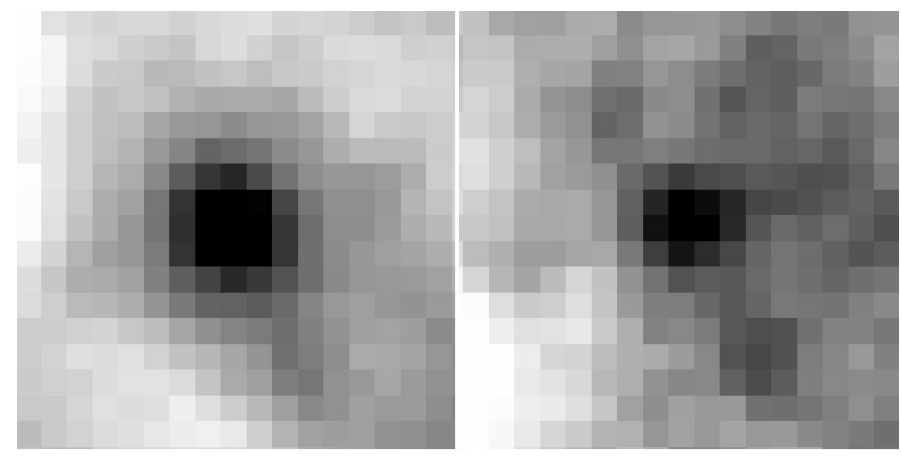

Fig. 6. Left: maps of extinction $(48 \times 48$ arcmin) obtained by averaging the maps centred on each cluster from the Abell sample. Right: the same restricted to those clusters at redshift $z<0.05$.

clusters at redshift $z<0.05$, the number of clusters selected is small (86 clusters). The stacked map (Fig. 6) shows a central excess of extinction $E(B-V)=(1.22 \pm 0.35) \times 10^{-3} \mathrm{mag}$, and then a flux density and luminosity of $(6.6 \pm 1.9) \times 10^{4} \mathrm{Jy} \mathrm{sr}^{-1}$ and $(0.12 \pm 0.01) \times 10^{45} \mathrm{erg} \mathrm{s}^{-1}$, respectively. For the sample at $z<0.05$, the profile has a $F W H M \sim 15$ arcmin, and a mean redshift 0.036 . Assuming Gaussian profiles as a rough approximation, and that the instrumental resolution is the one given by the profile obtained for the Wen et al. clusters (i.e. $F W H M \sim 12$ arcmin, see next section), this gives a mean angular extension of the dust emission in Abell clusters at redshifts $<0.05$ of $\sim 9$ arcmin, which corresponds to a radius of $\sim 0.6 \mathrm{Mpc}$.

\subsection{Comparison with previous works}

\subsubsection{Method 1}

Comparison between our results and those found by other authors are not straightforward, due to the different methods and samples used and the different spatial scales proved. In general, all the modern works pointed out to a reddenig effect $<$ few $10^{-3}$ mag of extinction per cluster. Our method relies on the hypothesis that the dust does not extend to distances larger than $6 \mathrm{Mpc}$ (or at least that it is not distributed uniformly on such scales). With all these cautions, our results are compatible with those previous works by Nollenberg et al. (2003), Chelouche et al. 2007, Bovy et al. (2008), and Muller et al. (2008), who present upper limits or marginal detections of excess at the level of $10^{-3} \mathrm{mag}$. However, the work by McGee \& Balogh (2010) explores the presence of dust on very large scales by measuring colour excess of QSOs behind 70000 low redshift SDSS galaxy groups and clusters. They claim the detection of dust out to a clustercentric distance of $30 \mathrm{Mpc} h^{-1}$. Although those authors measured the excess of colours in very large scales (tens of Mpc), their results (see Fig. 4 in their paper) indicate an excess of colour in $g-i \sim 5 \times 10^{-3} \mathrm{mag}$, which is difficult to reconcile with the radial profile of that excess, as it is shown in Fig. 3. We do not have an explanation for that inconsistency.

\subsubsection{Method 2}

As mentioned earlier, the only previous statistical work using a similar method was conducted by Montier \& Giard (2005). These authors found an average central emission at $100 \mu \mathrm{m}$ of $(3.40 \pm 0.14) \times 10^{4} \mathrm{Jy} / \mathrm{sr}$, whilst we obtained $(1.88 \pm 0.08) \times$ $10^{4} \mathrm{Jy} / \mathrm{sr}$. A direct comparison between both amplitudes is not possible because we need to take into account the different

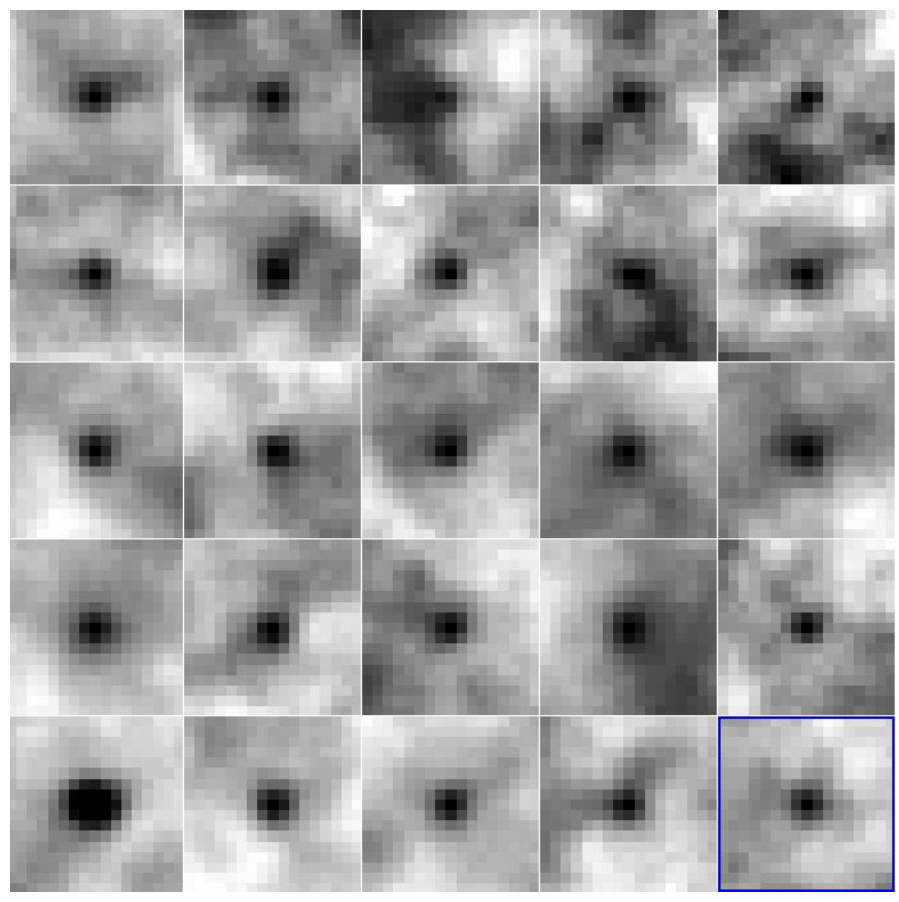

Fig. 7. Map of extinction $(48 \times 48$ arcmin $)$ obtained averaging the maps centred on each cluster. Each map corresponds to a given range in richness and redshift. Richness increases from top to bottom, while redshift increases from left to right. The mean values of richness and redshift in each bin are those quoted in Table 2.

angular width of both dust profiles. The FWHM of their distribution at $100 \mu \mathrm{m}$ has a $F W H M \sim 8 \operatorname{arcmin}$ (see their Fig. 5), whilst we obtained (see previous section) a $F W H M \sim 12 \operatorname{arcmin}$. By assuming two dimensional Gaussian profiles, the dilution factor, $(12 / 8)^{2}=2.25$, between both angular distributions and the ratio of the peak amplitudes (1.8) translates into a flux density $\sim 25 \%$ in our case. This relative agreement is quite remarkable considering the different samples of clusters used. The reasons for our comparatively wider profile must come from the use of the Schlegel et al. maps with a $F W H M \sim 6.1$ arcmin instead of the IRIS maps (Miville-Deschenes \& Lagache 2005) with a resolution $F W H M \sim 4.3$ arcmin used by Montier \& Giard. Additionally, some extra widening in our distribution could be due to the several improvements of IRIS maps and in particular the better destripping. This could also account for the reason that the relative uncertainties in both studies are similar despite the fact of the higher number of clusters we have used.

\subsection{Dependence with richness and/or redshift}

To determine the origin and possible evolution of dust in clusters, it is interesting to study the possible dependence of the dust emission with respect to mass and/or redshift. This was done by splitting the clusters in $5 \times 5$ bins. The limits were chosen to have roughly the same number $\left(\sim 2 \times 10^{3}\right)$ clusters in each bin. The masses, $M_{200}$, were obtained from the relation between richness and mass given by Wen et al. (their Eq. (2)). Although the resulting maps (Fig. 7) are relatively noisy as compared to the stacked map of the full sample presented in Fig. 4, all the maps show a clear detection of signal with the maximum at the centre of the maps. The main problem having a low number of clusters is the existence of gradients or systematics in the extinction map that prevents for a good estimation of the level of Galactic extinction. The analysis of the beam profiles indicate that they are 
C. M. Gutiérrez and M. López-Corredoira: On the dust of galaxy clusters

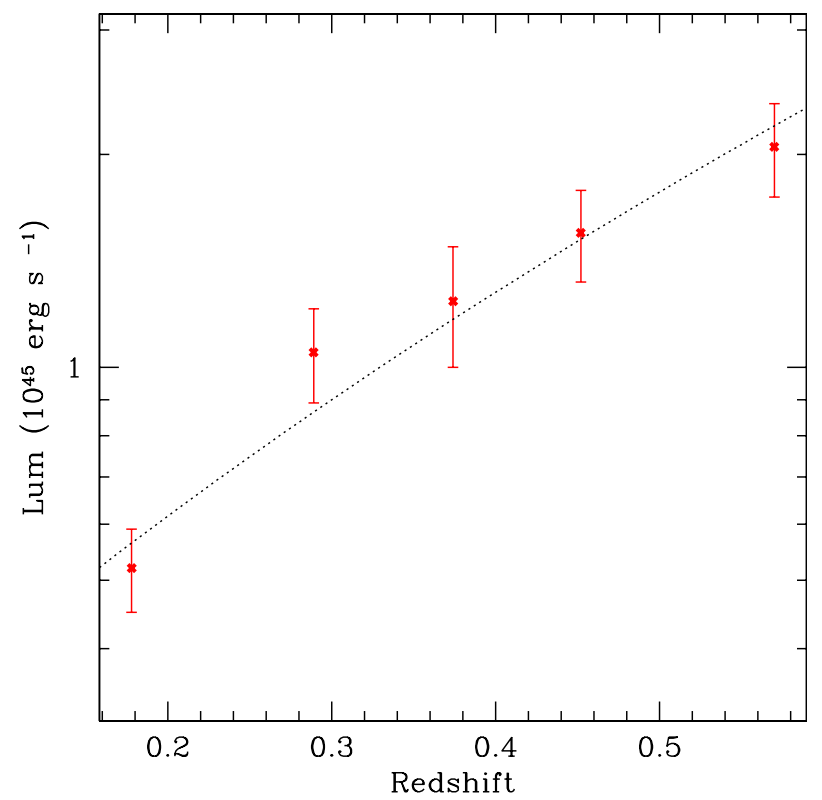

Fig. 8. Estimated mean cluster luminosity at $100 \mu \mathrm{m}$ versus redshift. The dotted line corresponds to a fit $L=L_{o}(1+z)^{\alpha}$ with $\alpha=4.73$.

dominated by the instrumental resolution and that the differences between them can be adscribed to the effect of noise and in particular to the uncertainties in estimating the background level. After doing many tests, we conclude that the best estimation of the luminosities in this case is obtained from the amplitude of the central peak when a Gaussian profile is assummed. This width was obtained from the mean value and rms of the distribution of the $5 \times 5$ profiles. We obtain $F W H M=11.7 \pm 1.9$ arcmin. The luminosities estimated according to the procedure outlined above are quoted in Table 2 . The horizontal and vertical axis represent redshift and mass, respectively. The five divisions in redshift correspond to central values (from left to right) $0.19,0.30$, $0.38,0.46$, and 0.57 , whilst the five vertical divisions correspond to mean masses of $(0.63,0.74,0.91,1.15$ and 2.2$) \times 10^{14} M_{\odot}$, respectively. There is a clear tendency to increase luminosity with redshift and richness. This was quantified by fitting a function $L=L_{0} \times(1+z)^{\alpha} \times\left(M_{\text {cluster }} / 10^{14} M_{\odot}\right)^{\beta} \mathrm{erg} \mathrm{s}^{-1}$. The best fit corresponds to values $\log \left(L_{0}\right)=44.41 \pm 0.10, \alpha=4.7 \pm 0.8$ and $\beta=0.64 \pm 0.17$. The evolution found with redshift agrees with the models by Le Floch'h et al. $(2005 ; \alpha=3.15 \pm 1.6)$ and Bay et al. $\left(2007 ; \alpha=4.0_{-2.2}^{+2.1}\right)$. Binning the maps in five bins in redshift and computing the luminosities in a similar way, we obtained the results presented in Table 3. As the five bins in redshift have roughly similar mean masses, we can ignore that dependence and fit a function $L=L_{0} \times(1+z)^{\alpha}$. The results are presented in Table 3 and Fig. 8. The luminosity found for the Abell clusters (see Sect. 3.3) at redshift $<0.05$ is $(0.12 \pm 0.01) \times 10^{45} \mathrm{erg} \mathrm{s}^{-1}$ (not included in the plot) which follows the general tendency to decline luminosity at lower redshifts. The ratio from the mean luminosity at redshift 0.57 (last bin in Table 3) to the mean luminosty of the Abell clusters is a factor $\sim 17$. Assuming that this ratio is entirely due to evolution in redshift (there could be some effect due to differences in richness), this confirms the high evolution in redshift found by other authors using large samples (e.g. Giard et al. 2008) or single clusters (Bay et al. 2007). These authors found a ratio $\sim 17$ between the luminosities at $24 \mu \mathrm{m}$ of Coma $(z=0.02)$ and MS1054-0321 $(z=0.83)$.
Table 2. Cluster luminosities at $100 \mu \mathrm{m}$ as a function of redshift and mass.

\begin{tabular}{cccc}
\hline \hline$N_{\text {clust }}$ & Mass $\left(10^{14} M_{\odot}\right)$ & $z$ & $L\left(10^{45} \mathrm{erg} \mathrm{s}^{-1}\right)$ \\
\hline 1773 & 0.633 & 0.189 & $0.50 \pm 0.17$ \\
1772 & 0.632 & 0.297 & $0.59 \pm 0.20$ \\
1845 & 0.632 & 0.382 & $0.54 \pm 0.32$ \\
2047 & 0.633 & 0.462 & $1.16 \pm 0.43$ \\
1574 & 0.634 & 0.574 & $1.13 \pm 0.50$ \\
2234 & 0.742 & 0.186 & $0.33 \pm 0.11$ \\
2081 & 0.739 & 0.297 & $0.94 \pm 0.34$ \\
2276 & 0.740 & 0.381 & $0.99 \pm 0.38$ \\
2635 & 0.740 & 0.463 & $0.93 \pm 0.37$ \\
2290 & 0.744 & 0.576 & $1.69 \pm 0.56$ \\
2321 & 0.911 & 0.184 & $0.48 \pm 0.17$ \\
2231 & 0.913 & 0.298 & $0.81 \pm 0.31$ \\
2337 & 0.914 & 0.381 & $1.38 \pm 0.53$ \\
2637 & 0.913 & 0.463 & $1.87 \pm 0.66$ \\
2690 & 0.916 & 0.583 & $2.33 \pm 0.80$ \\
2056 & 1.153 & 0.184 & $0.61 \pm 0.20$ \\
1736 & 1.154 & 0.297 & $1.11 \pm 0.39$ \\
1868 & 1.152 & 0.380 & $1.32 \pm 0.49$ \\
2012 & 1.148 & 0.463 & $2.11 \pm 0.75$ \\
2189 & 1.150 & 0.586 & $1.98 \pm 0.71$ \\
2712 & 2.407 & 0.180 & $0.75 \pm 0.25$ \\
1995 & 2.249 & 0.296 & $1.57 \pm 0.54$ \\
1852 & 2.121 & 0.379 & $2.17 \pm 0.73$ \\
1879 & 1.994 & 0.462 & $1.86 \pm 0.65$ \\
1615 & 1.940 & 0.584 & $2.91 \pm 1.00$ \\
\hline
\end{tabular}

Table 3. Cluster luminosities at $100 \mu \mathrm{m}$ as a function of redshift.

\begin{tabular}{cccc}
\hline \hline$N_{\text {clust }}$ & Mass $\left(10^{14} M_{\odot}\right)$ & $z$ & $L\left(10^{45} \mathrm{erg} \mathrm{s}^{-1}\right)$ \\
\hline 9944 & 1.246 & 0.178 & $0.52 \pm 0.07$ \\
10297 & 1.146 & 0.289 & $1.05 \pm 0.16$ \\
9722 & 1.098 & 0.374 & $1.23 \pm 0.24$ \\
10739 & 1.046 & 0.452 & $1.55 \pm 0.23$ \\
11955 & 1.043 & 0.570 & $2.05 \pm 0.31$ \\
\hline
\end{tabular}

\section{Conclusions}

The main conclussions of our work are

- We have conducted a study to estimate the amount and distribution of dust within galaxy clusters. This has been done following two methods: (i) analyzing the effect that such dust produces on the light of objects in their background; and (ii) analyzing the contribution of clusters to the $E(B-V)$ extinction map by Schlegel et al. (1998).

- We did not find evidence of additional reddening of background galaxies with respect to galaxies in the field. Our analysis imposes maximum limits in the excess of colour due to intracluster dust extinction $\int_{0}^{R} \Delta(g-i) \mathrm{d} S=-3.67 \pm 3.52$, $+3.20 \pm 6.77$ and $-4.53 \pm 10.10$ milimags $\mathrm{Mpc}^{2}$ within clustercentric distances of 1,2 and $3 \mathrm{Mpc}$ respectively.

- Using the second method, we clearly detect the far infrared emission produced by the clusters. The corresponding extinction profile can be characterized by a Gaussian function with a peak amplitude of $346 \times 10^{-6}$ mag and a $F W H M \sim$ 12 arcmin. The angular profile is dominated by instrumental effects due to the resolution of the extinction map and the method used and does not reflect the spatial distribution of the dust within the clusters. Averaging the extinction of all clusters, we find a surface integral of the excess of colour $g-i$ of 3.4 millimag $\mathrm{Mpc}^{2}$. 
- The above extinction corresponds to an average flux and luminosties at $100 \mu \mathrm{m}$ per cluster of $0.21 \mathrm{Jy}$ and $(1.46 \pm 0.03) \times 10^{45} \mathrm{erg} \mathrm{s}^{-1}$, respectively. This signal can be explained as due to emission of $2 \times 10^{9} M_{\odot}$ of dust with temperature of $20 \mathrm{~K}$.

- Our results do not allow us to exclude the existence of some intracluster dust, but we constrain the maximum amount of dust to be a few tens the dust in Milky Way-like galaxies.

- Separating the clusters in $5 \times 5$ bins in redshift and richness respectively, we found a clear detection in each of them. Fitting a function $L=L_{0} \times(1+z)^{\alpha} \times$ $\left(M_{200} / 10^{14} M_{\odot}\right)^{\beta} \mathrm{erg} \mathrm{s}^{-1} \mathrm{erg} \mathrm{s}^{-1}$. The best fit corresponds to values $\log \left(L_{0}\right)=44.41 \pm 0.10, \alpha=4.7 \pm 0.8$, and $\beta=0.64 \pm 0.17$. The dependence in redshift agrees with previous studies.

Despite the results of previous studies and the work presented here, new techniques exploring the dust content in galaxy clusters for different subsamples of objects covering different ranges in mass and redshift are needed. Among the tools that can provide a deeper insight in the topic we would like to mention those allowing a whole treatment of dust and gas and the use of the recent maps obtained by the Planck/Herschel mission. As we have shown, the extinction due to dust in the intracluster media is too small to be measured with current datasets of galaxies but can potentially be of interest once new dataset are available.

Acknowledgements. Thanks are given to the anonymous referee for helpful comments. Funding for SDSS-III has been provided by the Alfred P. Sloan Foundation, the Participating Institutions, the National Science Foundation, and the US Department of Energy Office of Science. The SDSS-III web site is http://www.sdss3.org/. SDSS-III is managed by the Astrophysical Research Consortium for the Participating Institutions of the SDSS-III Collaboration including the University of Arizona, the Brazilian Participation Group, Brookhaven National Laboratory, Carnegie Mellon University, University of Florida, the French Participation Group, the German Participation Group, Harvard University, the Instituto de Astrofisica de Canarias, the Michigan State/Notre Dame/JINA Participation Group, Johns Hopkins University, Lawrence Berkeley National Laboratory, Max Planck Institute for Astrophysics, Max Planck Institute for Extraterrestrial Physics, New Mexico State University, New York University, Ohio State University, Pennsylvania State University, University of Portsmouth, Princeton University, the Spanish Participation Group, University of Tokyo, University of Utah, Vanderbilt University, University of Virginia, University of Washington, and Yale University. We have used also the NED (NASA Extragalactic Database, http: //nedwww.ipac.caltech.edu/)

\section{References}

Ahn, C. P., Alexandroff, R., Allende Prieto, C., et al. 2012, ApJS, 203, 21

Bai, L., Marcillac, D., Rieke, G. H., et al. 2007, ApJ, 664, 181

Berlind, A. A., Frieman, J., Weinberg, D. H., et al. 2006, ApJS, 167, 1

Bogart, R. S., \& Wagoner, R. V., 1973, ApJ, 181, 609

Bovy, J., Hogg, D. W., \& Moustakas, J. 2008, ApJ, 688, 198

Boyle, B. J., Fong, R., \& Shanks, T. 1988, MNRAS, 231, 897

Chelouche, D., Koester, B. J., \& Boewn, D. V. 2007, ApJ, 671, L97

Cox, P., Kruegel, E., \& Mezger, P.G. 1986, A\&A, 155, 380

Davies, J. I., Trewhella, M., Jones, H., et al. 1997, MNRAS, 288, 679

Draine, B. T. 1990, The Interstellar Medium in Galaxies, eds. H. A. Thronson Jr., \& J. M. Schull (Dordrecht: Kluwer), 483

Draine, B. T., \& Salpeter. E. E. 1979, ApJ, 231, 77

Drimmel, R., \& Spergel, D. N. 2001, ApJ, 556, 181

Giard, M., Montier, L., Pointecouteau, E., \& Simmat, E. 2008, A\&A, 490, 547

Gladders, M. D., \& Yee, H. K. C. 2005, ApJS, 157,1

Karanchetsev, I. D., \& Lipovetskii, V. A. 1969, Soviet Astron., 12, 909

Kelly, D. M., \& Rieke, G. H. 1990, ApJ, 361, 354

Kitayama, T., Ito, Y., Okada, Y., et al. 2009, ApJ, 695, 1191

Koester, B. P., McKay, T. A., Annis, J., et al. 2007, ApJ, 660, 239

Le Floc'h, E., Papovich, C., Dole, H., et al. 2005, ApJ, 632, 169

Loeb, A., \& Haiman, Z. 1997, ApJ, 490, 571

López-Corredoira, M., Cabrera-Lavers, A., Gerhard, O. E., \& Garzón, F. 2004, A\&A, 421, 953

Maoz, D. 1995, ApJ, 455, L115

Mathis, J. S. 1990, ARA\&A, 28, 37

McGee, S. L., \& Balogh, M. L. 2010, MNRAS, 405, 2069

Miville-Deschênes, M.-A., \& Lagache, G. 2005, ApJS, 157, 302

Montier, L. A., \& Giard, M. 2005, A\&A, 439, 35

Muller, S., Wu, S.-Y., Hsieh, B.-C., et al. 2008, ApJ, 680, 975

Myers, A. D., Outram, P. J., Shanks, T., et al. 2003, MNRAS, 342, 467

Nollenberg, J. G., Williams, L. L. R., \& Maddox, S. J. 2003 ApJ, 125, 2927

Romani, R. W., \& Maoz, D. 1992, ApJ, 386, 36

Roncarelli, M., Pointecouteau, E., Giard, M., Montier, L., \& Pello, R. 2010, A\&A, 512, A20

Schlafly, E. F., \& Finkbeiner, D. P. 2011, ApJ, 737, 103

Schlegel, D. J., Finkbeiner, D. P, \& Davis, M. 1998, ApJ, 500, 525

Schneider, D. P., Hall, P. B., Richards, G. T., et al. 2007, AJ, 134, 102

Stickel, M., Klaas, U., Lemke, D., \& Mattila, K. 2002, A\&A, 383, 367

Wen, Z. L., \& Han, J. L. 2013, The Intriguing Life of Massive Galaxies, Proc. International Astronomical Union, IAU Symp., 295, 188

Wen, Z. L., Han, J. L., \& Liu, F. S. 2012, ApJSS, 199, 34

Wickramasinghe, N. C., \& Wallis, D. H. 1996, Ap\&SS, 240, 157

Wise, M. W., O'Connell, R. W., Bregman, J. N., \& Roberts, M. S. 1993, ApJ, 405,94

Xilouris, E., Alton, P., Alikakos, J., et al. 2006, ApJ, 651, 107

Zwicky, F. 1962, in, Problems of Extragalactic research, ed. G. C. McVittie (NY: Macmillan), 149 